\title{
A Hybrid RANS-LES Computational Fluid Dynamics Simulation of an FDA Medical Device Benchmark
}

\author{
Primož DREŠAR*, Jožef DUHOVNIK** \\ *University of Ljubljana, Faculty of Mechanical Engineering, Aškerčeva 6, 1000 Ljubljana, Slovenia, \\ E-mail: primoz.dresar@lecad.fs.uni-lj.si \\ **University of Ljubljana, Faculty of Mechanical Engineering, Aškerčeva 6, 1000 Ljubljana, Slovenia, \\ E-mail: jozef.duhovnik@lecad.fs.uni-lj.si
}

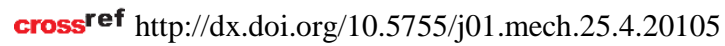

\section{Introduction}

Computational fluid dynamics (CFD) does not yet enjoy complete confidence in the field of medical devices development. For this reason, the United States Food and Drug Administration (FDA) has initiated a program to address this issue and to help the development of the applied CFD technology in the evaluation of medical devices [1]. A simple nozzle with a throat and sudden expansion has been introduced (Fig. 1) as a benchmark. It is a generalized representation of the flow through various devices in medicine. Multiple research groups have conducted experiments with transparent fluid analogous to blood material properties and a refractive index to match surrounding acrylic housing [13]. Using such an approach, the particle image velociometry (PIV) investigation became viable, and the velocity fields on the cross planes of the device could be examined. Such experimental data can be used for validation of turbulence models in the development of different devices and applications in medicine $[4,5]$. Obtaining accurate turbulence flow solutions still presents a great challenge in CFD. The most accurate and most expensive in terms of computer resources and time is the Direct Numerical Simulation (DNS), which is too prohibitive for industrial flows with a moderate to very complex wall-bounded geometries and high Reynolds numbers $(R e)$ flows. A Large Eddy Simulation (LES) model that filters some of the smallest eddies and resolves large ones has already been published for the nozzle benchmark [6]. The latter investigation showed excellent agreement with experimental data. Additionally, different research groups reported that various Reynolds-averaged NavierStokes (RANS) turbulence models showed larger discrepancies towards the experiments [7, 8]. Since the LES method has high requirements regarding the mesh topology and number of finite volume (FV) cells, which significantly lengthen the necessary time to get a solution, there are more economical approaches. Hybrid RANS-LES turbulence models are one such alternative [8]. They combine the best of both worlds with the use of special blending functions that switch between LES and RANS depending on when the mesh topology complies with the restrictions for a specific turbulence model. For instance, if the mesh is sufficiently refined, the flow can be resolved in LES mode, otherwise it uses one of the standard RANS models $(k-\varepsilon, k-\omega$, Shear Stress Transport, etc.) in the regions where the flow is statistically modeled. In this study several turbulence models are examined: a Detached Eddy Simulation (DES) [9-11] and its successor a Stress Blended Eddy Simulation (SBES) [12], both using high resolution (HR) and blended central difference (BCD) advection schemes. In addition, a Scale Adaptive Simulation (SAS) [13, 14], an advanced RANS model case, is computed for a comparison. All investigated hybrid models use a standard Shear Stress Transport (SST$\mathrm{k} \omega$ ) model [15], when in RANS mode. A commercial FVbased solver ANSYS CFX (Ansys Inc., Canonsburg, PA, USA) code was used to conduct a series of simulations with a Reynolds number of $R e=6500$ (at throat diameter), which represents a fully turbulent flow.

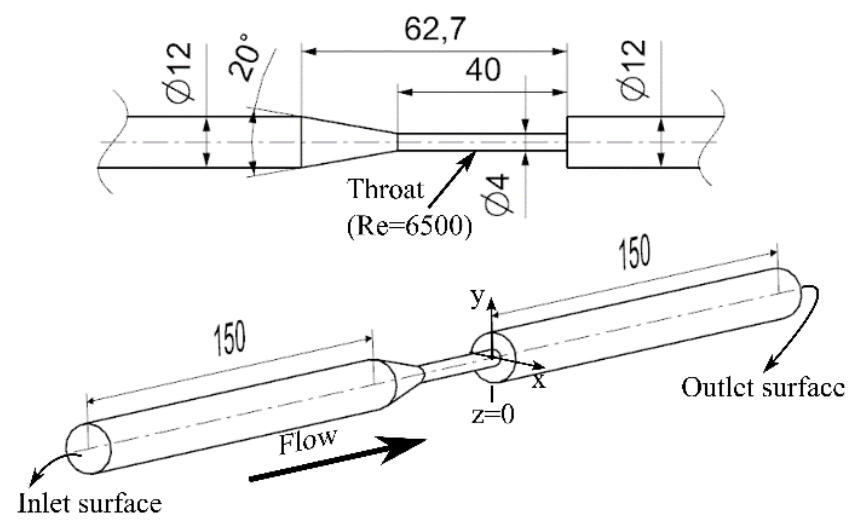

Fig. 1 A geometric representation of the numerical domain of FDA nozzle benchmark

\section{Materials and methods}

\subsection{D model and mesh preparation}

The 3D geometry consists of a tube with a throat and sudden expansion along the $z$-axis (Fig. 1). The numerical domain is built entirely of hexahedron elements using Ansys ICEM-CFD software (Ansys Inc., Canonsburg, PA, USA). The O-grid method is used to form layered structured elements in the near wall region with a first element height of $\sim 0.007 \mathrm{~mm}$. The layered element thickness in near-wall regions grows away from the walls with the expansion factor of 1.2 (Fig. 2). In our structured grid, high regularity of elements is met. The lowest Jacobian parameter for the hexahedron element regularity is 0.8 in the finest, and 0.5 in coarsest mesh, respectively.

\subsection{Physical properties and computation settings}

The fluid material setup in this device simulates blood with Newtonian fluid properties. The computations were conducted using an isothermal and incompressible 
fluid setup with 1 bar of reference pressure. A dynamic viscosity was set to $3.5 \mathrm{mPa} \cdot \mathrm{s}$, and a density of $1056 \mathrm{~kg} / \mathrm{m}^{3}$ was prescribed. On average, an advanced turbulence computation (DES, SAS, and SBES) has run $\sim 3$ days on a cluster of 20 computer nodes containing 24 Intel Xeon E5-2680 2.5 GHz processors with $256 \mathrm{~GB}$ DDR4 RAM. The SST-k $\omega$ computation took $\sim 1.5$ days using the same computer resources. A non-dimensional wall distance, $\mathrm{y}^{+}$of less than 1 was achieved in all cases. The time step (TS) for the transient simulations was set to $4 \cdot 10^{-5} \mathrm{~s}$ for a total case running time of $1 \mathrm{~s}$. The basis for the chosen TS lies in its relationship to Courant-Friedrichs-Lewy (CFL) condition. Consequently a root mean square (RMS) CFL number was kept below 1 and with it a sufficient level of the numerical convergence was obtained. For a time discretization, a secondorder backward Euler scheme was set for all computational cases.

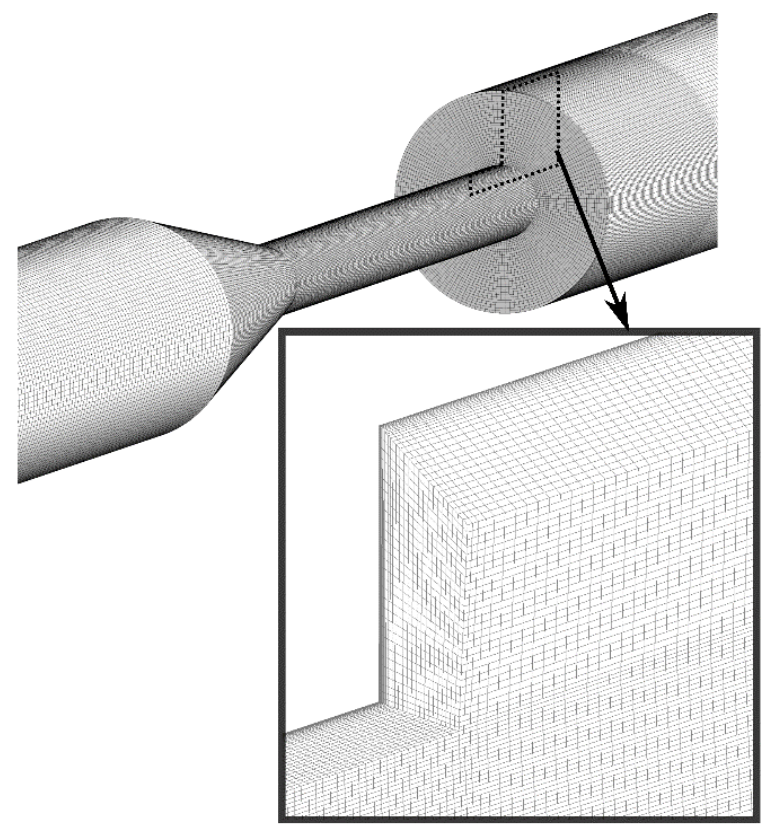

Fig. 2 All-structured hexahedron mesh representation of a numerical nozzle model

\subsection{Boundary and initial conditions}

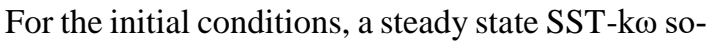
lution was used. At the nozzle binding walls, a no-slip wall boundary condition (BC) was applied. For the inlet condition a $1 / 7^{\text {th }}$ power law velocity profile was assumed [16]. It is an approximation for turbulent velocity profile, expressed as:

$$
V(r)=V_{\max }\left(1-\frac{r}{R_{\max }}\right)^{1 / 7}
$$

where: $r$ is the variable radius in the direction outward from the centerline of the nozzle, $R_{\max }$ is the maximal radius of the pipe, and $V_{\max }$ is the velocity at the centerline of the noz- zle, located at the inlet. Average velocity $\left(V_{a v e}\right)$ was computed from the Reynolds number $R e=6500$ at the throat cross-section (Fig. 1). The relationship between average and maximum velocities is calculated as:

$$
V_{\text {ave }}(r)=\frac{V_{\max }}{R_{\max }} \int_{0}^{R_{\max }}\left(1-\frac{r}{R_{\max }}\right)^{1 / 7} d r=\frac{7}{8} V_{\max } .
$$

At the outlet surface $\mathrm{BC}$, a relative pressure of $0 \mathrm{~Pa}$ was defined.

\section{The governing equations}

A fundamental challenge of CFD is finding a solution of discrete Navier-Stokes equations. As previously mentioned, our investigation covers two different hybrid turbulence models concepts. The first two models (delayedDES and SBES) are based on the DES approach and the second is a SAS method. All use the SST-k $\omega$ formulation for RANS modeling. SST- $\mathrm{k} \omega$ is the two-equation eddy-viscosity RANS model introduced by Menter [15]. It is a combination of the $k-\omega$ and $k-\varepsilon$ models [17]. In the fluid domain, better performance of the $k$ - $\omega$ model is closer to the wall since it has a good near wall modelling properties. $k-\varepsilon$ performs better in free-stream regions of the flow. SST switches between the two models with the use of special blending functions $\left(F_{1}\right.$ and $\left.F_{2}\right)$. A more profound definition and background can be found in the literature [15].

\subsection{A short definition of SAS-SST (2007)}

The SAS-SST model [12-14] consists of $k$ and $\omega$ equations (derived from SST model) with an extra $Q_{S A S}$ term (Eq. (5)). It is an advanced unsteady-RANS model that is capable of exhibiting LES-like behavior. The transport equation for turbulence kinetic energy $k$, is given by:

$$
\begin{aligned}
& \frac{\partial \rho k}{\partial t}+\frac{\partial}{\partial x_{j}}\left(\rho \bar{U}_{j} k\right)= \\
& =P_{k}-\rho c_{\mu} k \omega+\frac{\partial}{\partial x_{j}}\left[\left(\mu+\frac{\mu_{t}}{\sigma_{k}}\right) \frac{\partial k}{\partial x_{j}}\right],
\end{aligned}
$$

where: $x_{j}$ are coordinates $(j=1,2,3), \bar{U}_{j}$ are averaged velocity components, $\rho$ is fluid density, $k$ is turbulent kinetic energy, $P_{k}$ is the turbulence kinetic energy production term, $\mu$ is the dynamic viscosity of the fluid, $\mu_{t}$ is the turbulent dynamic viscosity, and $\omega$ is the specific dissipation rate. The transport equation for $\omega$ is denoted:

$$
\begin{aligned}
& \frac{\partial \rho \omega}{\partial t}+\frac{\partial}{\partial x_{j}}\left(\rho \bar{U}_{j} \omega\right)=\alpha \frac{\omega}{k} P_{k}-\rho \beta \omega^{2}+Q_{S A S}+ \\
& +\frac{\partial}{\partial x_{j}}\left[\left(\mu+\frac{\mu_{t}}{\sigma_{\omega}}\right) \frac{\partial \omega}{\partial x_{j}}\right]+\left(1-F_{1}\right) \frac{2 \rho}{\sigma_{\omega 2}} \frac{1}{\omega} \frac{\partial k}{\partial x_{j}} \frac{\partial \omega}{\partial x_{j}} .
\end{aligned}
$$

The SAS-SST special term is given as:

$$
Q_{S A S}=\max \left[\rho \zeta_{2} \kappa S^{2}\left(\frac{L}{L_{v K}}\right)^{2}-C \frac{2 \rho k}{\sigma_{\Phi}} \max \left(\frac{1}{\omega^{2}} \frac{\partial \omega}{\partial x_{j}} \frac{\partial \omega}{\partial x_{j}}, \frac{1}{k^{2}} \frac{\partial k}{\partial x_{j}} \frac{\partial k}{\partial x_{j}}, 0\right)\right]
$$


where:

$$
L_{v K}=\kappa \frac{S}{\left|\sqrt{\frac{\partial^{2} \bar{U}_{i} \partial^{2} \bar{U}_{i}}{\partial x_{k}{ }^{2} \partial x_{j}{ }^{2}}}\right|},
$$

and

$$
L=\sqrt{k} /\left(c_{\mu}^{1 / 4} \omega\right)
$$

$L_{v K}$ is the von Karman length scale, $L$ is length scale of modeled turbulence, and $S$ is a scalar invariant of the strain rate tensor. The SAS-SST turbulence model constants are: $\kappa, C, C_{\mu}, \beta, \zeta_{2}, \sigma_{k}, \sigma_{\omega 2}$, and $\sigma_{\Phi}[12]$. The $k$ and $\omega$ equations (eq. 3 and 4 ) in this formulation are actually modified SST model equations. The $Q_{S A S}$ term increases its value if enough unsteadiness in the flow is present, which results in decreased turbulent viscosity. In this way, it can produce LES-like solutions in unstable flow regions. At the same time, it uses RANS modeling capabilities where the flow is stable.

\subsection{DES-SST turbulence formulation}

The DES model is a hybrid RANS-LES model [911] that uses special blending functions to switch from RANS to the LES model in regions where the turbulent length scale $L_{t}$ from RANS model is larger than local mesh spacing $\Delta_{\max }=\max (\Delta x, \Delta y, \Delta z)$. The model used in our simulation is a version combined with the SST model and introduced by Strelets [18]. The length scale $L_{t}$ used in the computation of the dissipation rate $\varepsilon$ in the $k$-equation is replaced by $\Delta_{\max }$. A dissipation rate term from the SST equation with DES modification is defined as $\varepsilon=\beta^{*} k \omega F_{D E S}$, where:

$$
\begin{aligned}
& F_{D E S}=\max \left(\frac{L_{t}}{C_{D E S} \Delta_{D E S}}\left(1-f_{p}\right), 1\right), \\
& L_{t}=\frac{k^{3 / 2}}{\varepsilon}=\frac{\sqrt{k}}{\beta^{*} \omega} .
\end{aligned}
$$

$L_{t}$ is the turbulent length scale, $\beta^{*}$ is coefficient, $\omega$ is specific dissipation rate, and $C_{D E S}$ is a model constant. Subsequently, the modified $k$-equation can be written as:

$$
\frac{\partial \rho k}{\partial t}+\frac{\partial}{\partial x_{j}}\left(\rho \bar{U}_{j} k\right)=P_{k}-\rho \frac{k^{3 / 2}}{\min \left(L_{t}, C_{D E S} \Delta_{\max }\right)}+\frac{\partial}{\partial x_{j}}\left[\left(\mu+\frac{\mu_{t}}{\sigma_{k}}\right) \frac{\partial k}{\partial x_{j}}\right]
$$

where: $\begin{aligned} & \left(C_{D E S} \Delta_{\max } \leq L_{t}\right) \rightarrow L E S \\ & \left(C_{D E S} \Delta_{\max }>L_{t}\right) \rightarrow R A N S\end{aligned}$

A modified blending function $f_{p}$ for a delayed-DES (DDES) formulation is enabled in our computation and is defined by:

$$
f_{p}=f_{D D E S}=1-\tanh \left[\left(C_{d 1} r_{d}\right)^{C_{d 2}}\right]
$$

where: $C_{d 1}$ and $C_{d 2}$ are model constants. The parameter $r_{d}$ is defined as:

$$
r_{d}=\frac{v+v_{t}}{\kappa^{2} d_{w}{ }^{2} \sqrt{0.5\left(S^{2}+\Omega^{2}\right)}}
$$

where: $\kappa$ is the von Karman constant, $d_{w}$ is wall distance, $v$ is a molecular viscosity, $v_{t}$ is RANS viscosity, $S$ and $\Omega$ are the magnitude of the strain rate and the magnitude of the vorticity, respectively.

\subsection{SBES-SST turbulence formulation}

The Stress-Blended Eddy Simulation (SBES) superseded both the delayed-DES (DDES) and shielded-DES (SDES) formulations [12]. In the SBES-SST model, a modified mesh spacing from SDES is used and is given by:

$$
\Delta_{S D E S}=\max \left(\sqrt[3]{V_{\text {cell }}}, 0.2 \Delta_{\max }\right)
$$

where: $V_{\text {cell }}$ is the volume of the cell. Additionally, the model coefficient is changed to $C_{D E S}=0.4$. A shielding function of a SDES model, $f_{s}$ achieves a blending on the stress level between RANS and LES formulations in the SBES model, as shown in an equation:

$$
\tau_{i j}^{S B E S}=f_{S} \tau_{i j}^{R A N S}+[1-f] \tau_{i j}^{L E S} .
$$

The function $f_{s}$. offers an asymptotic shielding under sufficient mesh refinement, and it has not yet been published. It is proprietary to Ansys Inc. The SBES turbulence model is in essence not an entirely new formulation, but rather a new way of blending between LES and RANS methods.

\section{Results}

\subsection{Grid independence test (GIT)}

A study of grid sensitivity was performed with six different meshes, and a time and area averaged pressure parameter was calculated on a cross plane at location $z=0.12$ $\mathrm{m}$. These results are presented in Fig. 3. The mesh with $\sim 6.5$ million elements was used for all subsequent computations (Table 1) since the investigated parameter achieved an asymptotic value at this mesh density.

Table 1

A number of mesh cells $\left(N_{H}\right)$ with computed averaged pressure results $\left(p_{\text {avg }}\right)$

\begin{tabular}{|l|l|l|l|l|l|l|}
\hline & Mesh 1 & Mesh 2 & Mesh 3 & Mesh 4 & Mesh 5 & Mesh 6 \\
\hline$N_{H}[-]$ & 501,566 & $1,509,113$ & $3,078,950$ & $4,524,282$ & $6,542,944$ & $7,526,172$ \\
\hline$p_{\text {avg }}[\mathrm{mmHg}]$ & -4.096 & -0.949 & 0.111 & 0.248 & 0.253 & 0.247 \\
\hline
\end{tabular}




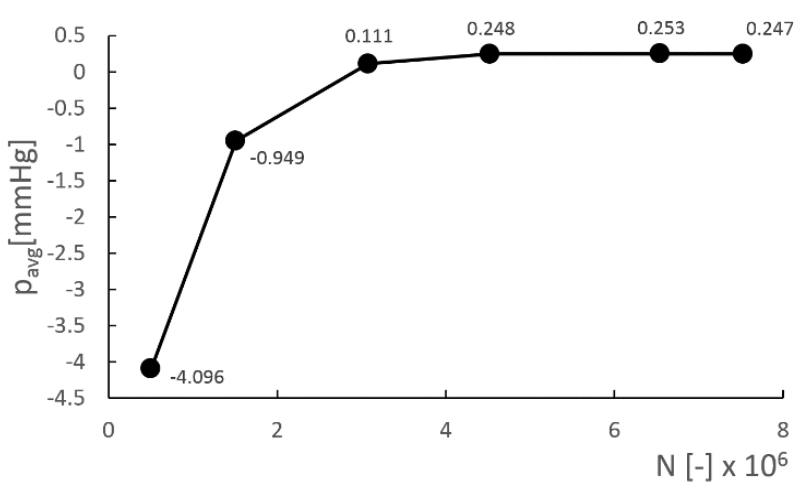

Fig. 3 A grid independence test performed on six meshes

4.2. Instantaneous velocity and flow structures representation

A velocity plot on a $2 \mathrm{D}$ longitudinal mid-plane (Fig. 4) shows instantaneous velocity magnitude contours on the left side (Fig. 4, a), and $Q$-criterion plots of the same results on the right side (Fig. 4, b). $Q$-criterion is a tool for $3 \mathrm{D}$ flow structures visualization, and is defined as a second invariant of the velocity gradient tensor [19]. A represented iso-surfaces of a $Q$-criterion surround locations where rotation dominates over the strain rate if the condition $Q>0$ is met. $Q$-criterion defined by:

$$
\begin{aligned}
& Q=0.5\left(\Omega_{i j} \Omega_{i j}-S_{i j} S_{i j}\right), \\
& \Omega_{i j}=0.5\left(\frac{\partial U_{i}}{\partial x_{j}}-\frac{\partial U_{j}}{\partial x_{i}}\right), \quad S_{i j}=\frac{1}{2}\left(\frac{\partial U_{i}}{\partial x_{j}}+\frac{\partial U_{j}}{\partial x_{i}}\right) .
\end{aligned}
$$

In this way a monitoring of scale resolved flow structures can be observed. Fig. 5 shows representation of flow on a longitudinal mid plane in the parameter of the eddy viscosity ratio. It is defined as a ratio between turbulent viscosity and molecular viscosity. It represents the amount of statistically generated viscosity, which is another indicator of scale-resolving capabilities.

$$
\begin{aligned}
& \text { Velocity magnitude }[\mathrm{m} / \mathrm{s}]
\end{aligned}
$$

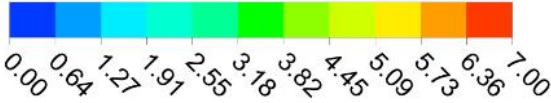

SBES-SST (BCD)

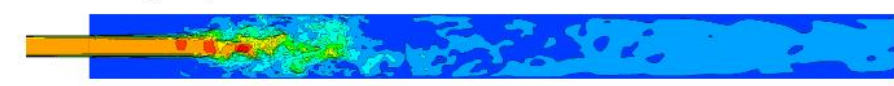

SBES-SST (HR)

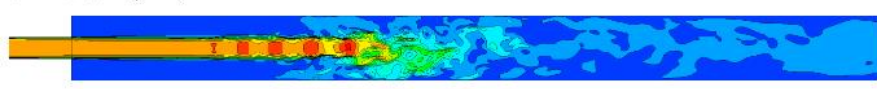

DDES-SST (BCD)

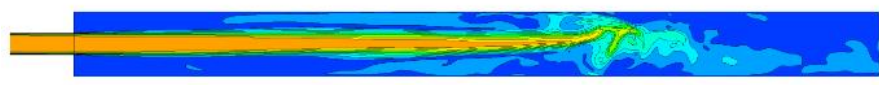

DDES-SST (HR)

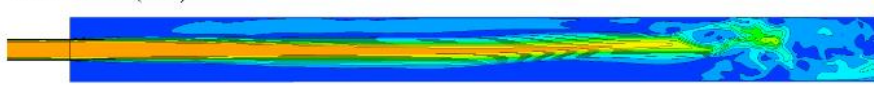

SAS-SST (BCD)

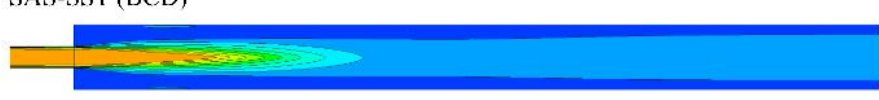

SST-ko (unsteady)

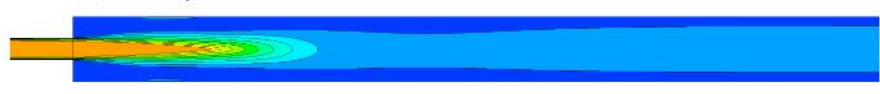

a

\section{$\mathrm{Q}=1000 \mathrm{~s}^{-2}$}
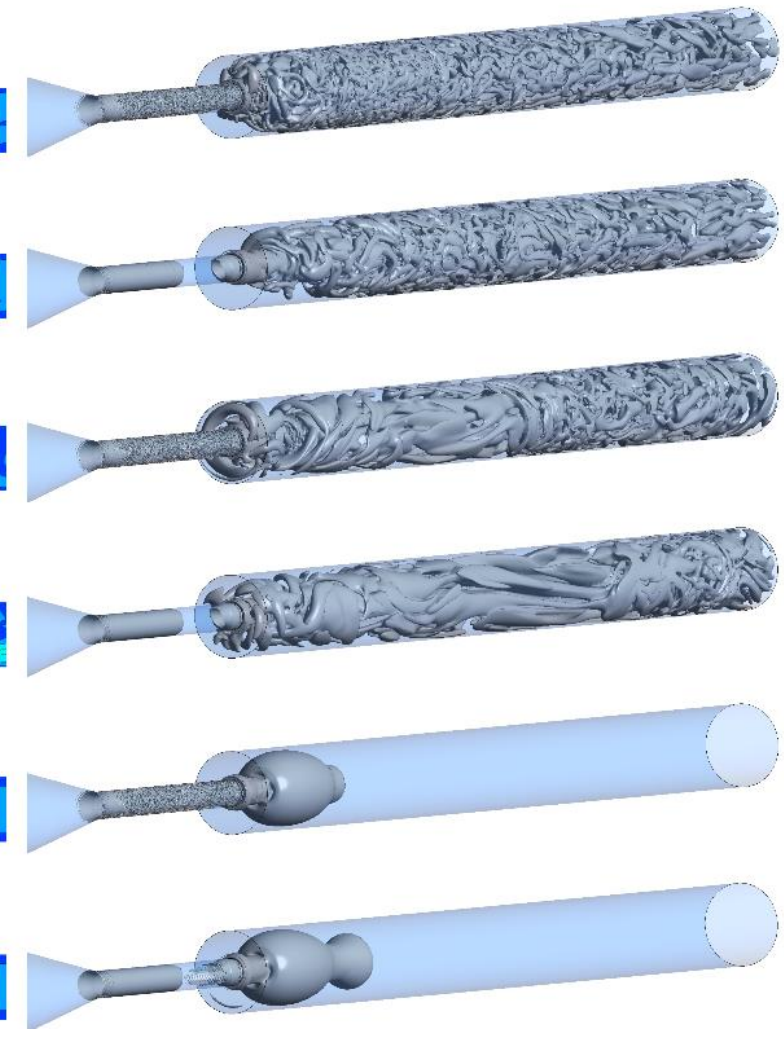

b

Fig. 4 Instantaneous velocity (a) and $Q$-criterion plots for analysed turbulence models (b)

\subsection{Validation of numerical simulations}

Our computational methods are validated by already published experimental data available online [20]. The data consist of results obtained from four independent laboratories. The profiles are located at locations on the $\mathrm{z}$ axis: $0 \mathrm{~m}, 0.008 \mathrm{~m}, 0.032 \mathrm{~m}$, and $0.06 \mathrm{~m}$ (Fig. 6). Velocity magnitude is time averaged. Velocity profiles at $z=0 \mathrm{~m}$ are located directly at sudden expansion and are coinciding with the coordinate system origin.

\section{Discussion}

A single solution case of the SAS-SST model can be pointed out (Fig. 4), which exhibits only a RANS-mode solution. SAS failed to produce any resolved features in the flow for the reason that local flow instabilities through the nozzle were too weak for the SAS model to trigger scale resolving (SR) capabilities, and it remained in the RANS mode. In this aspect, SAS is less suitable for this application than DESbased models. Both RANS solutions SAS and SST models 
exhibited ten times larger magnitude of turbulent viscosity ratio in the flow than the rest of the SR models (Fig. 5), which is another indicator that the simulation runs in RANS mode. A $Q$-criterion plot, as expected, exhibited undeveloped flow structures with the SST and SAS-SST models (Fig. 4, b). Conversely, both DES-based models showed resolved coherent flow structures (Fig. 4, b). Enabling the BCD advection scheme also showed an improvement in solution over the HR scheme. A HR scheme achieved RMS residual of the main computed equation below $1 \times 10^{-5}$ in all cases, whereas the BCD scheme had a momentum equation RMS residual about an order of magnitude higher. The finest vorticity structures can be observed using the DES-based methods combined with BCD scheme (Fig. 4, b). Axial velocity plots (Fig. 6) show that all models have similar and slightly under-predicted velocity profiles (Fig. 6, a) in com- parison to the experimental results. Fig. 6, b shows a backflow close to the walls with the SST and SAS models, and slight under-prediction of the bulk flow close to a centreline, for all models. The velocity profiles tend to differ among themselves on locations shown on Fig. 6, c and 6, d. In these two cross-sections, turbulence models with HR schemes tend to over-predict velocity profile. The SBES-BCD model provides the best agreement with the experiment at location $z=0.032 \mathrm{~m}$. The Delayed-DES model tends to have reattachment issues with both advection schemes (Fig. 6, d). At location $z=0.06 \mathrm{~m}$ the best results are shown by SST, SAS (in RANS mode), and SBES-BCD. On Fig. 7, an axial velocity in the throat region is slightly under-predicted, while after sudden expansion, all turbulence model curves tend to show diversity. The closest results compared to experimental data are SBES (BCD) and models running completely in RANS mode.

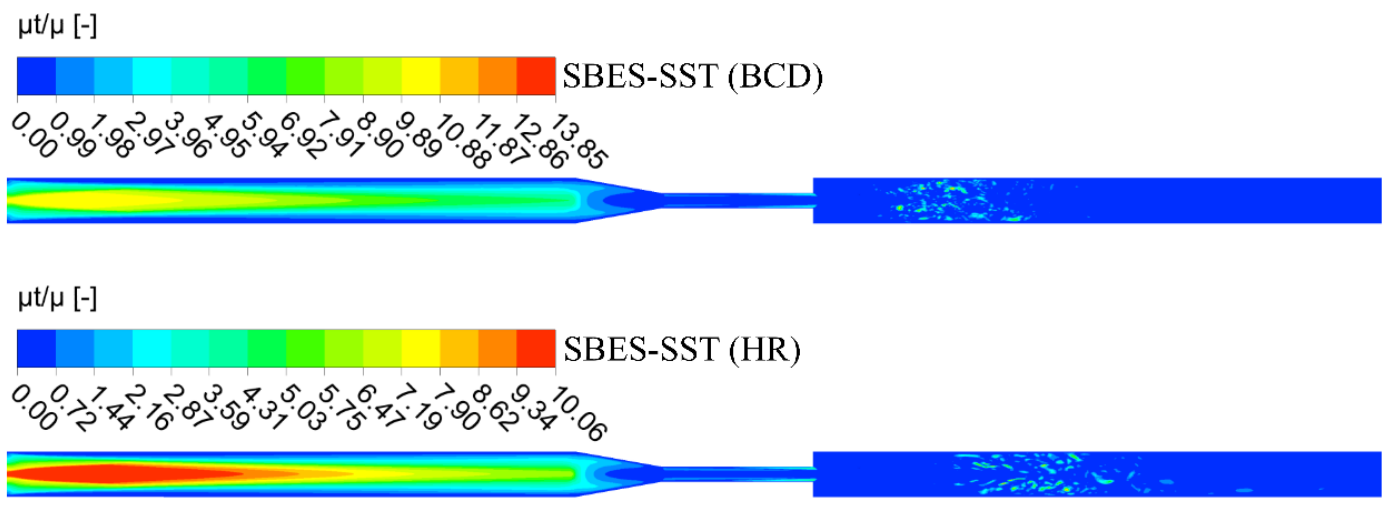

$\mu \mathrm{t} / \mu[-]$

DDES-SST (BCD)

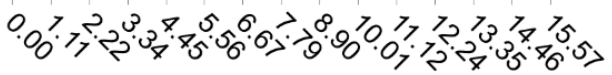

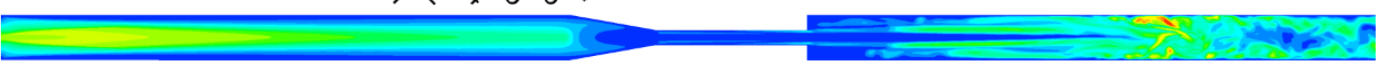

$\mu \mathrm{t} / \mu[-]$

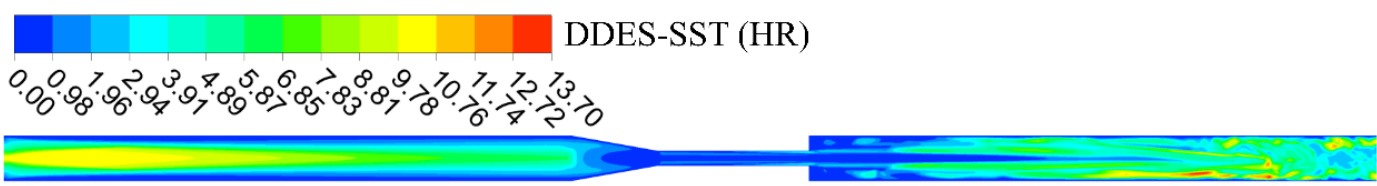

$\mu \mathrm{t} / \mu[-]$

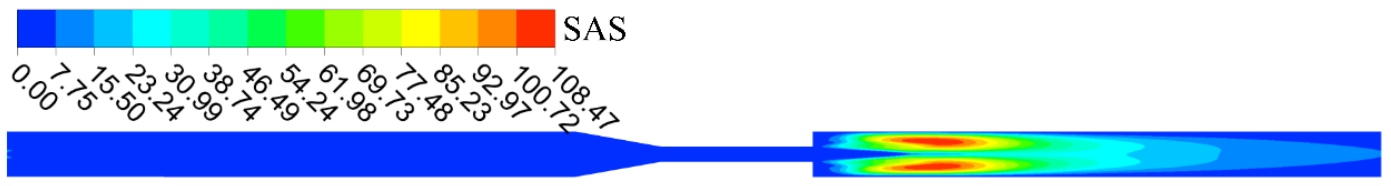

$\mu \mathrm{t} / \mu[-]$

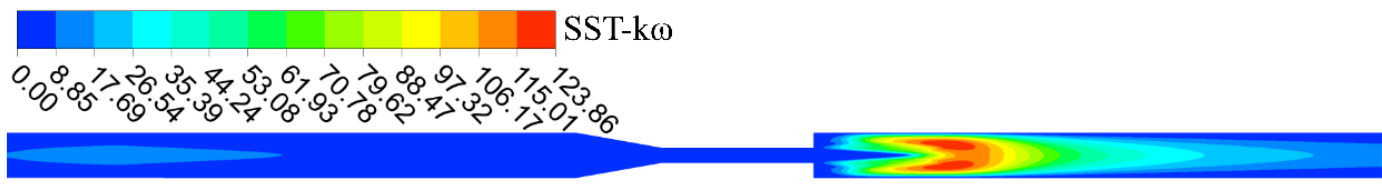

Fig. 5 Eddy viscosity ratio for all investigated turbulence model case 
- Exp. $Z=0 \mathrm{~m} \longrightarrow$ SBES_BCD ---SBES_HR

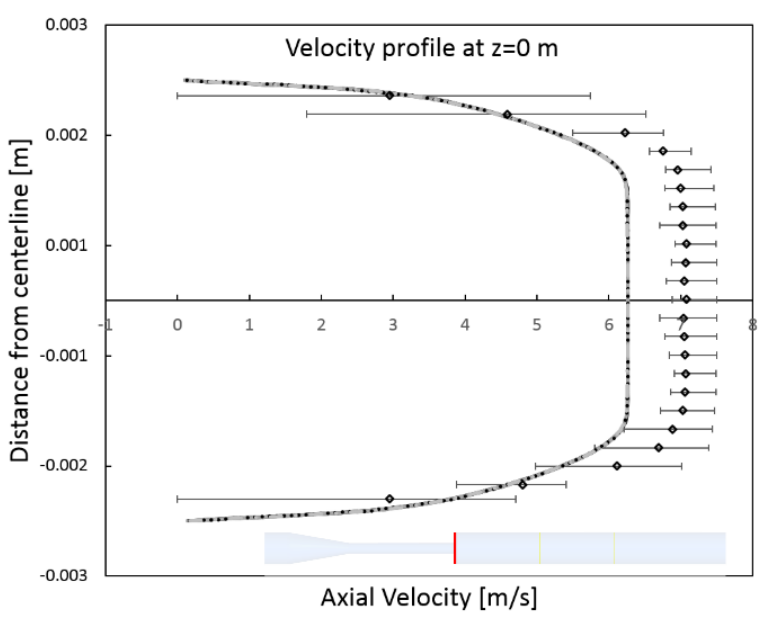

a

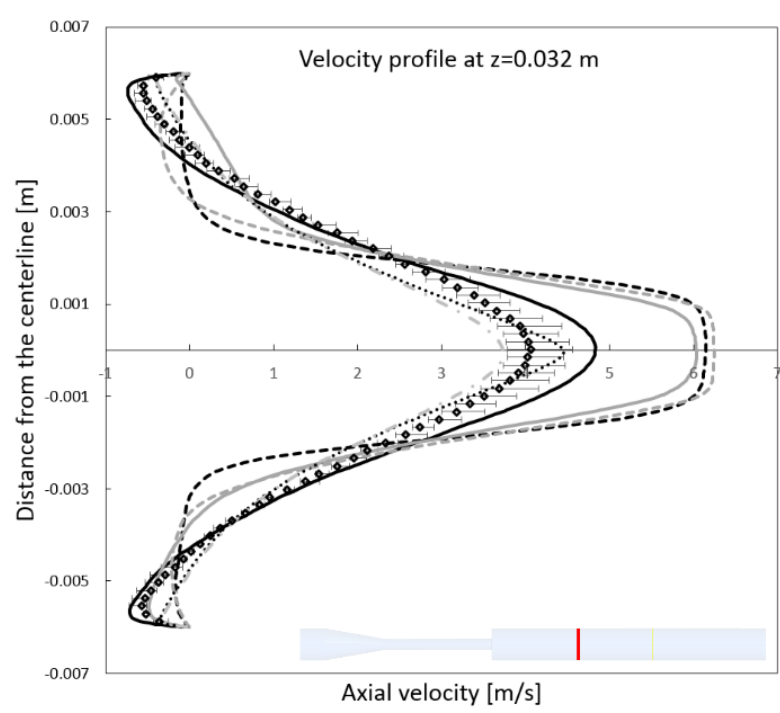

C
-DDES_BCD ---DDES_HR $\quad \cdots . .$. SAS $\quad-$ SST

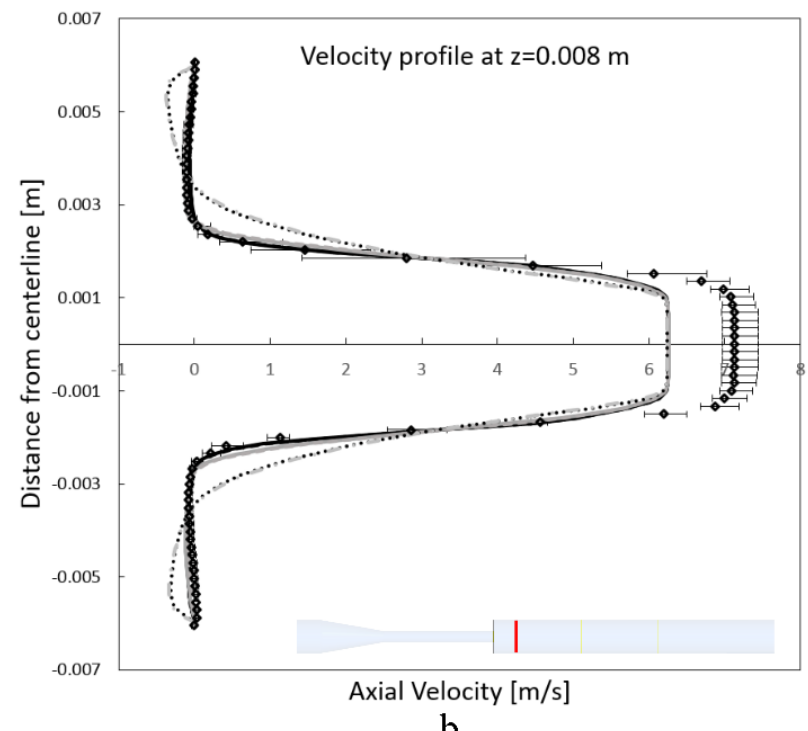

b

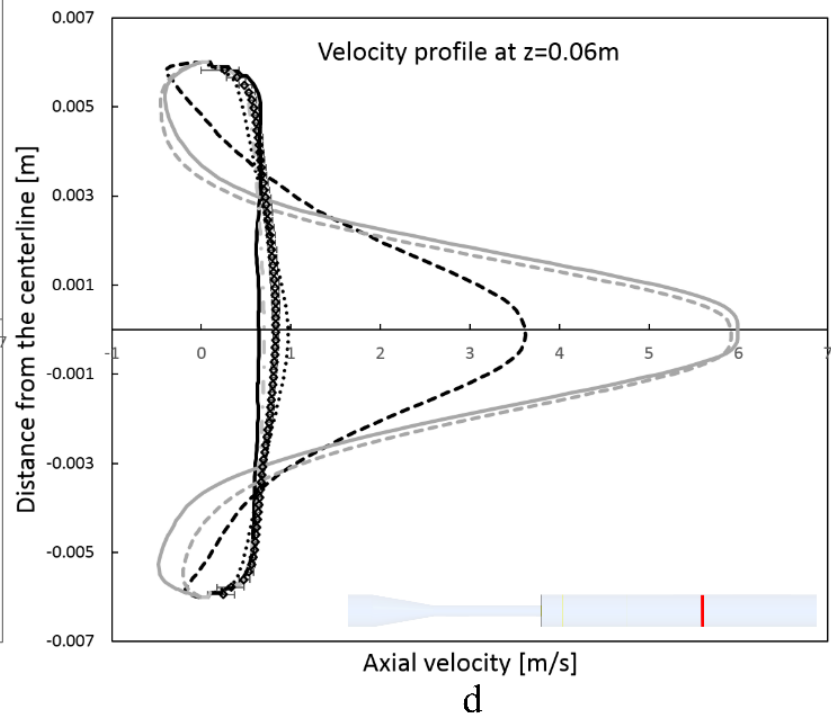

Fig. 6 Axial velocity profiles at four different locations on $z$-axis compared to the experimental data. Axial time-averaged velocity profiles are located at $z=0 \mathrm{~m}(\mathrm{a}), z=0.008 \mathrm{~m}(\mathrm{~b}), z=0.032 \mathrm{~m}$ (c) and $z=0.06 \mathrm{~m}(\mathrm{~d})$

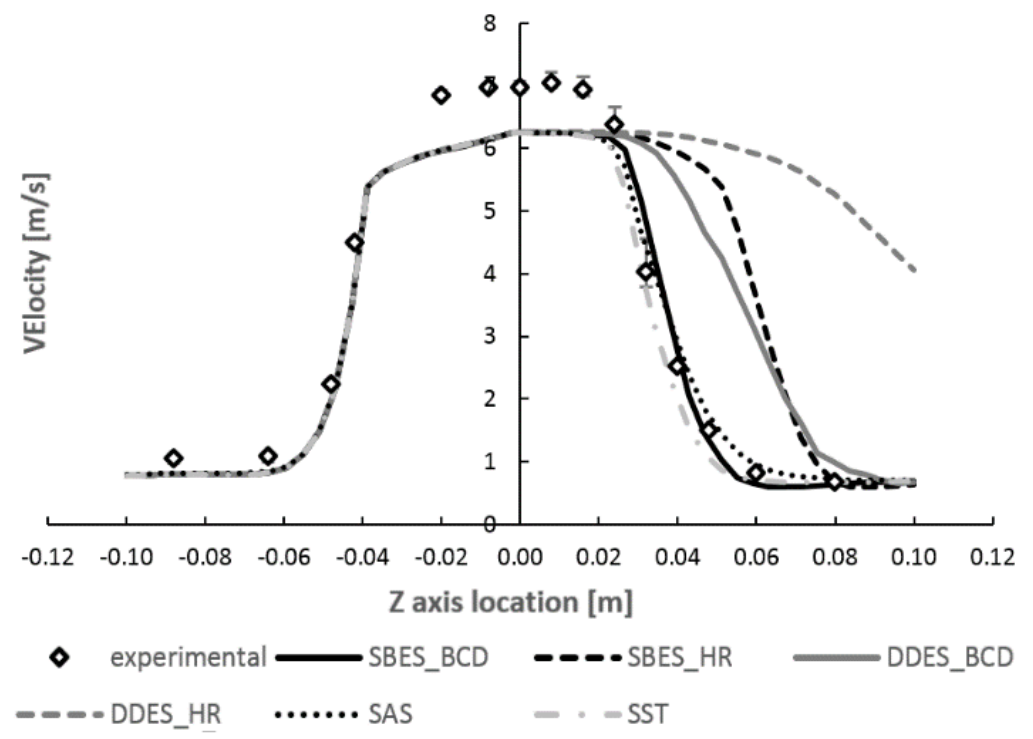

Fig. 7 A validation of axial velocity magnitude solution on the centreline, on a chosen interval 


\section{Conclusions}

This study fills the gap between strictly RANS and LES simulations. The use of LES models is still expensive in terms of computer resources and time in engineering applications. Therefore, LES-RANS hybrids are becoming more viable in complex wall-bounded flows at medium or high turbulent $R e$ numbers.

This investigation compared three formulations of RANS-LES hybrid turbulence models combined with two different advection schemes settings and validated them with already published experimental results. The results showed that this type of flow did not produce enough unsteadiness in the flow for the SAS-SST model to be triggered in SR mode. In general, the positive side of SAS model is that it is not affected by the grid refinement near the boundary layer and therefore a grid-induced separation of the flow does not pose an issue. In DES-based models that issue is present. So, a lot of effort was put into a development of blending functions that would shield a premature flow separation. Tackling this issue has continued since the early stages of DES model development. After all, the main goal of scale resolving or LES-RANS hybrid model is to model a boundary layer turbulence in RANS mode, and the mixing layer in the resolved mode. SBES-SST, with BCD mode enabled, achieves that best among the studied models, and is the closest in capabilities to LES model for a much lower cost.

\section{References}

1. Hariharan, P.; Giarra, M.; Reddy, V.; Day, S.; Manning, K. B.; Deutsch, S.; Stewart, S. F. C.; Myers, M. R.; Berman, M. R.; Burgreen, G. W.; Paterson, E. G. and Malinauskas, R. A. 2011. Multilaboratory particle image velociometry analysis of the FDA Benchmark nozzle model to support validation of computational fluid dynamics simulations, ASME J. Biomech. Eng. 133(4): 041002. http://dx.doi.org/10.1115/1.4003440.

2. Yano, T.; Funayama, M.; Sudo S.; Mitamura, Y. 2012. Analysis of flow within a left ventricle model fully assisted with continuous flow through the aortic valve, Artificial Organs, 36(8): 714-723.

http://dx.doi.org/10.1111/j.1525-1594.2012.01513.x. http://dx.doi.org/10.1115/1.1865190.

3. Day, W. S.; McDaniel, J. C. 2005. PIV measurements of flow in a centrifugal blood pump: time varying flow, ASME J. Biomech. Eng. 127: 254-263.

4. Ford, M. D.; Nikolov, H. N.; Milner, J. S.; Lownie, S. P.; DeMont, E. M.; Kalata, W.; Loth, F.; Holdsworth, D. W.; Steinman, D. A. 2008. PIVmeasured versus CFD-predicted flow dynamics in anatomically realistic cerebral aneurysm Models, ASME J. Biomech. Eng. 130(2): 021015. http://dx.doi.org/ 10.1115/1.2900724.

5. Day, S. W.; McDaniel, J. C. 2005. PIV measurements of flow in a centrifugal blood pump: Time-Varying Flow, ASME J. Biomech. Eng. 127: 254-263. http://dx.doi.org/ 10.1115/1.1865190. http://dx.doi.org/ 10.1016/j.compbiomed.2014.01.004.

6. Janiga, G. 2014. Large Eddy simulation of the FDA Benchmark nozzle for a reynolds number of 6500 , Computers in Biology and Medicine, 47: 113-119.
7 Stewart, S. F. C.; Paterson, E. G.; Burgreen, G. W., Hariharan, P.; Giarra, M.; Reddy, V.; Day, S. W.; Manning, K. B.; Deutch, S.; Berman, M. R.; Myers, M. R.; Malinauskas, R. A. 2012. Assessment of CFD performance in simulations of and idealized medical device: Results of FDA's First Computational Interlaboratory Study, Cardiovascular Engineering and Technology 3(2): 139-160. http://dx.doi.org/ 10.1007/s13239-012-0087-5.

8. Hariharan, P; D'Souza, P. A.; Horner, M.; Morrison, T. M.; Malinauskas, R. A.; Myers, R. M. 2016. Use of the FDA nozzle model to illustrate validation techniques in Computational Fluid Dynamics (CFD) Simulations, PLOS ONE: 1-25. A. Malinauskas, Matthew R. Myers. https://doi.org/10.1371/journal.pone.0178749.

9. Fröhlich, J.; Terzi, D. 2008. Hybrid LES/RANS methods for the simulation of turbulent flows, Progress in Aerospace Sciences 44(5): 349-377.

http://dx.doi.org/ 10.1016/j.paerosci.2008.05.001.

10. Spalart, P. R.; Deck, S.; Shur, M. L., Squires, K. D.; Sterlets, M. Kh.; Travin, A. 2006. A New version of Detached-Eddy simulation, resistant to ambiguous densities, Theor. Comput. Fluid Dyn. 20: 181-195. http://dx.doi.org/ 10.1007/s00162-006-0015-0.

11. Spalart, P. R. 2001. Young-Person's Guide to Detached-Eddy Simulation Grids. NASA CR-2001211032. https://ntrs.nasa.gov/archive/nasa/casi.ntrs.nasa.gov/20010080473.pdf.

12. ANSYS, 2017, CFX Solver Theory Guide 18.2.

13. Menter, F. R.; Egorov Y. 2010. The scale-adaptive simulation method for unsteady turbulent flow predictions, Part 1: Theory and Model Description. Flow, Turbulence and Combustion 85(1): 113-138.

http://dx.doi.org/ 10.1007/s10494-010-9264-5,

14. Menter, F. R.; Egorov Y.; Lechner R.; Cokljat D. 2010. The scale-adaptive simulation method for unsteady turbulent flow predictions. Part 2: Application to Complex Flows, Flow, Turbulence and Combustion 85(1): 139-165.

http://dx.doi.org/ 10.1007/s10494-010-9265-4.

15. Menter, F. R. 1994. Two-equation Eddy-Viscosity turbulence models for engineering applications, AIAA Journal 32(8): 1598-1605. http://dx.doi.org/ 10.2514/3.12149.

16. De Chant, L. J. 2005. The venerable $1 / 7$ th power law turbulent velocity profile: A classical nonlinear boundary value problem solution and its relationship to stochastic processes, Applied Mathematics and Computation 161: 463-474. http://dx.doi.org/ 10.1016/j.amc.2003.12.109.

17. Wilcox, D. C. 1994. Turbulence Modeling for CFD-2 ${ }^{\text {nd }}$ edition, DCW Industries, Inc., La Canada, California.

18. Sterlets, M. 2001. Detached Eddy simulation of massively separated flows, In Proceedings of the AIAA $39^{\text {th }}$ Aerospace Sciences Meeting, Reno, Nevada, USA, Paper 2001-0879. http://dx.doi.org/ 10.2514/6.2001-879.

19. Hunt, J. C. R.; Wray, A. A.; Moin, P. 1988. Eddies, stream and convergence zones in turbulent flows, In Proceedings of the Summer Program. NASA-Stanford Center for Turbulence Research. 
https://ntrs.nasa.gov/ar-

chive/nasa/casi.ntrs.nasa.gov/19198900151.pdf.

20. Standardization of Computational Fluid Dynamics (CFD) Techniques Used to Evaluate Performance and Blood Damage Safety in Medical Devices: An FDA Critical Path Initiative; Round-robin \#1', 2008, https://www.fda.gov/scienceresearch/specialtopics/criticalpathinitiative/spotlightoncpiprojects/ucm149414.htm.

\section{P. Drešar, J. Duhovnik}

\section{A HYBRID RANS-LES COMPUTATIONAL FLUID DYNAMICS SIMULATION OF AN FDA MEDICAL DEVICE BENCHMARK}

\section{S u m m a r y}

Computational fluid dynamics (CFD) is a valuable tool that complements experimental data in the developmentof medical devices. The reliability of CFD still presents an issue and for that reason, no standardized approaches are currently available. The United States Food and Drug Administration (FDA) has initiated the development of a program for CFD validation, and has presented an idealized nozzle benchmark model. In this study, a nozzle flow with sudden expansion has been simulated using advanced RANS-LES turbulence models. Such models partially resolve the flow and are cheaper in computer resources and time in comparison to the Large Eddy Simulation (LES). Furthermore, they are more accurate than standard Reynolds-averaged Navier-Stokes (RANS) models. A collection of hybrid turbulence models has been investigated: Detached Eddy Simulation (DES), Stress Blended Eddy Simulation (SBES), and Scale Adaptive Simulation (SAS), and compared to a standard RANS Shear Stress Transport (SST) model. Subsequently, all models were validated by experimental results already published by different research groups. Particle Image Velociometry (PIV) experiments were performed by interlaboratory study, and the results are available online for numerical validation. The flow conditions in this study are only restricted to a turbulence flow at a Reynolds number of $R e=6500$. Complementing the turbulence models investigation, two advection schemes were tested: high resolution (HR) and bounded central difference scheme (BCD). Among all advanced models the SBES model with BCD scheme has the best agreement with the experimental values.

Keywords: computational fluid dynamcis, turbulence, medical device benchmark, velocity fields.

Received February 05, 2018 Accepted August 26, 2019 